\title{
Deformidad de Madelung: Criterios actuales en radiografía simple
}

Dres. Horacio Saavedra $\mathbf{M}^{(1)}$, Daniel Ríos $\mathbf{Q}^{(2)}$, Jorge Díaz $\mathbf{J}^{(2)}$.

1. Becado de Radiología, Centro de Imagenología, Hospital Clínico Universidad de Chile. Santiago - Chile.

2. Radiólogo, Centro de Imagenología, Hospital Clínico Universidad de Chile. Santiago - Chile.

\section{Madelung deformity: current criteria in radiography}

Abstract: Madelung deformity is a common finding in various conditions affecting the wrist, with characteristic changes in the radius, carpus and ulna, the most characteristic being the triangular configuration of the carpal and the ulnar inclination of the distal radial epiphysis. It can be seen on simple radiography, computed tomography or magnetic resonance. As radiologists, we must know the currently recommended criteria, having it present for early diagnosis in young patients, or when the deformity is already established in older patients. Keywords: Deformity, Leri-Weill Disease, Radiology, X-ray of the wrist.

Resumen: La deformidad de Madelung constituye un hallazgo común de varias patologías que afectan a la muñeca, con alteraciones características en el radio, carpo y cúbito, siendo la configuración triangular del carpo y la inclinación cubital de la epífisis distal del radio los más característicos. Puede ser visto en radiografía simple, tomografía computada o resonancia magnética. Como radiólogos, debemos conocer los criterios recomendados en la actualidad, teniéndolos presentes para su diagnóstico precoz en pacientes jóvenes o cuando la deformidad ya está establecida en pacientes de edad más avanzada.

Palabras clave: Deformidad, Enfermedad de Léri-Weill, Radiología, Radiografía de muñeca.

Saavedra H, Ríos D, Díaz J. Deformidad de Madelung: Criterios actuales en radiografía simple. Rev Chil Radiol 2015; 21(1): 18-21.

Contacto: Dr. Horacio Saavedra / dr.hsaavedra@gmail.com

Trabajo recibido el 19 de noviembre de 2014. Aceptado para publicación el 25 de febrero de 2015.

\section{Introducción}

La deformidad de Madelung (DDM) se define como un trastorno del crecimiento de la porción anteromedial de la placa de crecimiento epifisiaria distal del radio ${ }^{(1)}, \mathrm{y}$ se atribuye clásicamente a una rara forma de displasia mesomélica caracterizada por la tríada de baja estatura, facie mongoloide y DDM, conocida como discondrosteosis de Léri-Weill. Sin embargo, también está presente en otras patologías que causan fusión prematura de la fisis distal del radio, como fracturas Salter-Harris tipo $\mathrm{V}^{(2)} \mathrm{O}$ artritis infecciosa radiocubital distal(3).

Las mujeres son más comúnmente afectadas que los varones. Puede ser bilateral en $50-66 \%$ de los pacientes. La deformidad no suele manifestarse hasta la adolescencia (10-14 años) $)^{(1)}$.

Habitualmente constituye un hallazgo que no produce mayor sintomatología ${ }^{(4)}$. Sin embargo, además de las consecuencias cosméticas, clínicamente puede presentarse con disminución de la fuerza de agarre, dolor en la muñeca en relación a la impactación ulnocarpiana y limitación del rango de movimiento que causa dificultades en las actividades cotidianas ${ }^{(1,5,6)}$. La sintomatología progresa en paralelo con el desarrollo puberal, pudiendo remitir espontáneamente en la adultez temprana o requerir cirugía. En los casos de remisión, puede manifestarse nuevamente asociado a cambios degenerativos en edades más avanzadas ${ }^{(4)}$.

\section{Aspecto imagenológico}

El cirujano alemán Otto Wilhelm Madelung en $1878^{(3)}$ describió la deformidad de la muñeca en base a hallazgos semiológicos, incluso antes de que Wilhelm Roentgen descubriera los rayos $X$ en 1895. Posteriormente, Dannenberg et al. ${ }^{(7,8)}$ en 1939 propuso doce criterios radiológicos que continúan enseñándose en los textos de radiología ${ }^{(8)}$ (Tabla I).

Basándose en los criterios de Dannenberg, Langer ${ }^{(9)}$ postuló que para hacer el diagnóstico deben estar al menos presentes los siguientes hallazgos radiográficos:

- Acortamiento del radio.

- Alteración de la forma cuadrangular de la epífisis distal radial que toma forma triangular.

- Configuración triangular de los huesos del carpo con el semilunar en el ápice, acuñándose entre la deformidad del radio y la protrusión del cúbito (Figura 1). 


\begin{tabular}{|l|}
\hline Tabla I \\
\hline Cambios en el Radio \\
\hline - Doble curvatura (medial y dorsal) \\
- Disminución de la longitud del hueso \\
- Forma triangular de la epífisis distal \\
- Fusión prematura de la parte medial de la fisis, \\
asociada a angulación medial y volar de la superficie \\
articular \\
- Zonas radiotransparentes focales a lo largo del borde \\
- Exedial del hueso \\
\hline Cambios en el Cúbito \\
- Subluxación dorsal \\
- Densidad aumentada (hipercondensación y \\
distorsión de la cabeza del cúbito) \\
\hline - Aumento de longitud del hueso \\
\hline Cambios en el Carpo \\
- Configuración triangular con el semilunar en el ápex \\
- Aumento de distancia entre el radio distal y el cúbito \\
\hline
\end{tabular}

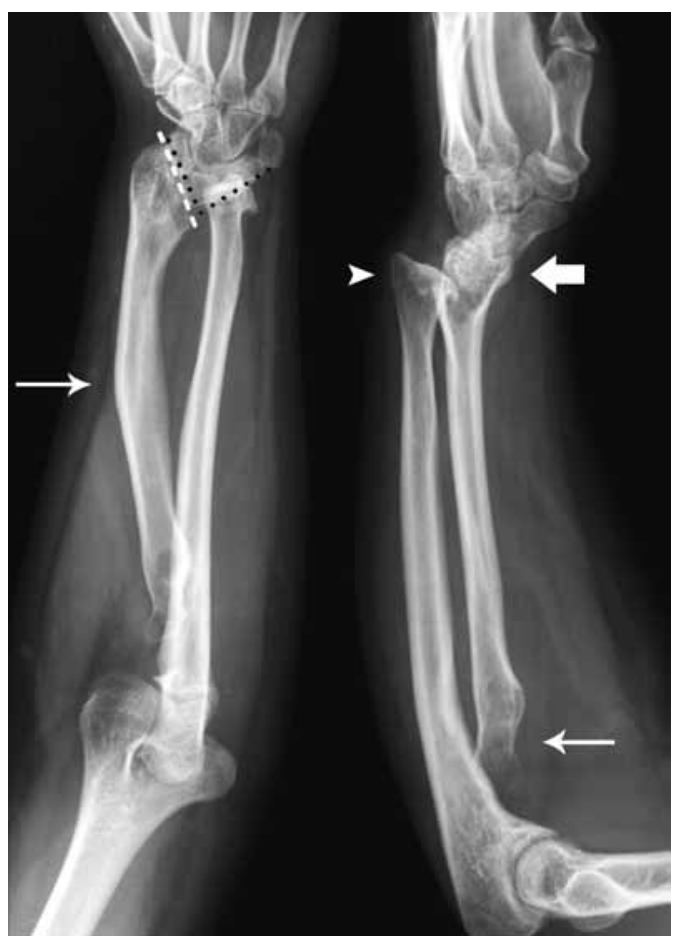

Figura 1. Deformidad de Madelung. Radiografía de antebrazo izquierdo frontal y lateral. Se observa un radio corto y curvo (flecha delgada) con mayor inclinación volar de su segmento distal (flecha gruesa), lo que determina un ensanchamiento de la articulación radio-cubital distal. Existe además una exagerada inclinación cubital de la superficie articular del radio distal (línea discontinua), con subluxación dorsal del cúbito (punta de fecha) y deformidad triangular del carpo (línea punteada). Los huesos del carpo forman una cuña en la deformidad triangular creada por el radio distal, con el semilunar en el ápex.
Los criterios clásicos expuestos hasta ahora describen detalladamente la DDM, sin embargo, éstos están presentes cuando la deformidad clínica es evidente.

En un intento por establecer un diagnóstico más precoz en ausencia de una deformidad obvia, McCarroll et al. ${ }^{(10-12)}$ estableció cuatro mediciones objetivas en radiografía simple. Sus criterios son los siguientes:

Inclinación cubital $\geq 33^{\circ}$ : En una radiografía $\mathrm{PA}$, la inclinación cubital se define como el complemento ( $90^{\circ}$-ángulo A) del ángulo agudo (ángulo A) formado entre el eje longitudinal del cúbito y una línea tangencial a las superficies proximales del escafoides y el semilunar (Figura 2).

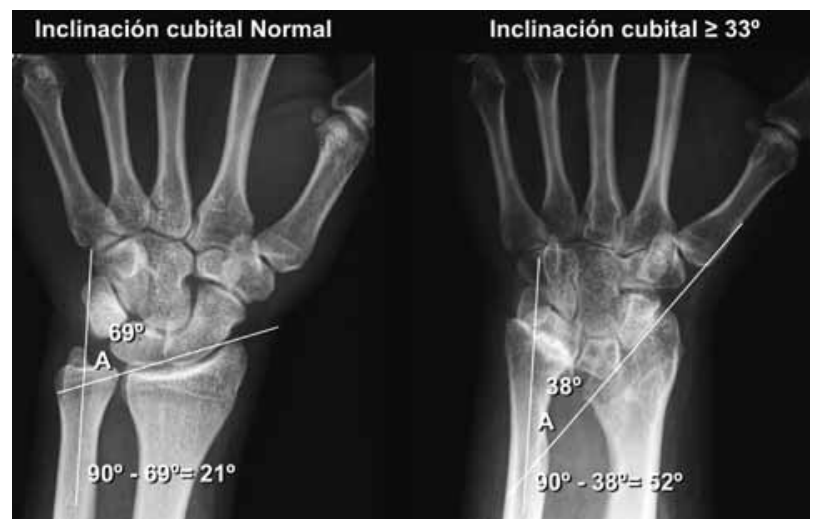

Figura 2. Inclinación cubital del carpo. Radiografía de muñeca izquierda $P A$. A la izquierda el ángulo $A$ tiene un valor de $69^{\circ}$ en un carpo normal, con una inclinación cubital de 21‥ A la derecha el ángulo A mide $38^{\circ}$, traduciendo una mayor inclinación cubital del carpo, alterada en la DDM.

Descenso semilunar $\geq 4 \mathrm{~mm}$ : En una radiografía $\mathrm{PA}$, el descenso semilunar se define como la distancia en milímetros (distancia $B$ ) entre el punto más proximal del semilunar y una línea perpendicular al eje longitudinal del cúbito y a través de su superficie articular distal. La medición es positiva si el cúbito se extiende distal a la superficie proximal del semilunar (Figura 3).

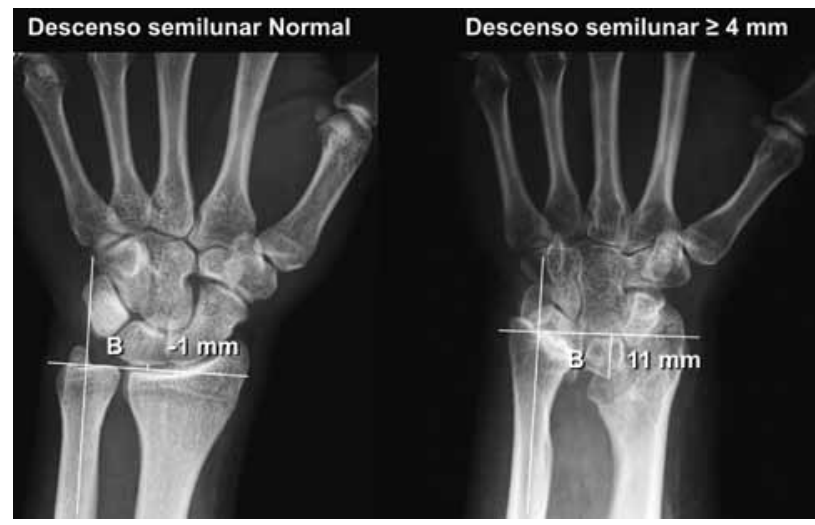

Figura 3. Descenso semilunar. Radiografía de muñeca izquierda $P A$. A la izquierda mediciones en un carpo normal, donde la distancia $B$ tiene un valor negativo, ya que el cúbito se extiende proximal al semilunar. A la derecha la distancia $B$ se encuentra alterada en la DDM. 
Ángulo de la fosa semilunar $\geq 40^{\circ}$ : En una radiografía PA, el ángulo de la fosa semilunar se define como el complemento ( $90^{\circ}$-ángulo $\left.\mathrm{C}\right)$ del ángulo agudo (ángulo $\mathrm{C}$ ) entre el eje longitudinal del cúbito y una línea a través de la fosa semilunar del radio (Figura 4).

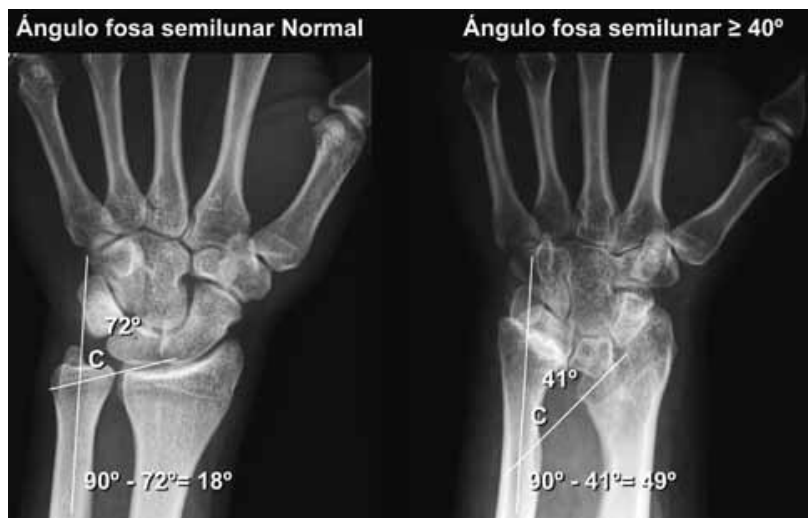

Figura 4. Ángulo de la fosa semilunar. Radiografía de muñeca izquierda $P A$. A la izquierda el ángulo $C$ tiene un valor de $72^{\circ}$, con un ángulo de la fosa semilunar de $18^{\circ}$ en un carpo normal. A la derecha ángulo C mide 41ํㅡㄹ determinando un ángulo de la fosa semilunar en rango patológico.

Desplazamiento volar del carpo $\geq 20 \mathrm{~mm}$ : En una radiografía lateral, el desplazamiento volar del carpo se define como la distancia en milímetros (distancia D) entre el eje longitudinal del cúbito y el punto más volar sobre la superficie del semilunar o del capitate (Figura 5).

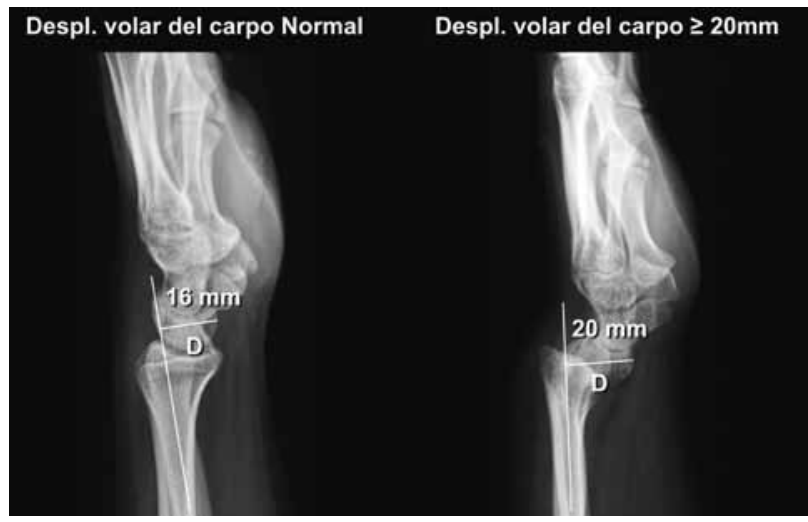

Figura 5. Desplazamiento volar del carpo. Radiografía de muñeca izquierda lateral. A la izquierda mediciones en un carpo normal, donde la distancia $D$ tiene un valor de 16 $\mathrm{mm}$. A la derecha la distancia $D$ se encuentra alterada en la DDM.

Basta sólo uno de los cuatro criterios presentes para establecer el diagnóstico de $\mathrm{DDM}^{(10)}$.

Otros métodos diagnósticos son la TC y RM. Mientras que los rayos $X$ (radiografía simple y tomografía computada) muestran la anatomía ósea detallada, las imágenes por resonancia magnética son muy útiles para ver los tejidos blandos incluyendo ligamentos, tendones y músculos. Así, puede verse la fisis anormal y la anatomía de los ligamentos en la cara palmar de la articulación radiocarpiana, identificando en los casos congénitos un ligamento engrosado emergiendo del margen cubital del radio distal hacia el semilunar, llamado ligamento de Vicker ${ }^{(13,14)}$.

El tratamiento es sintomático, incluyendo órtesis y rehabilitación. En casos de dolor y limitación de las actividades de la vida diaria, existen distintas técnicas quirúrgicas, incluyendo epifisiolisis del radio, resección del ligamento de Vicker y osteotomías ${ }^{(15)}$.

\section{Diagnóstico diferencial}

Aunque tras la descripción original de Madelung la deformidad fue atribuida principalmente a la discondrosteosis de Léri-Weill, hoy se acepta que ésta está presente en diversas patologías, pudiendo estar asociada a condiciones genéticas y displásicas (síndrome de Léri-Weill, síndrome de Turner, síndrome uña-rótula, exostosis hereditaria múltiple, enfermedad de Ollier, acondroplasia, mucopolisacaridosis de Hurler), causas secundarias (trauma, infección, insuficiencia vascular, trastornos musculares) o ser idiopática ${ }^{(5,6,16)}$. La DDM clásica es típicamente bilateral, asimétrica y más común en mujeres. Si se observa osteopenia y metacarpianos cortos, debemos pensar en el síndrome de Turner. Si la DDM es unilateral, dos causas comunes son el trauma y las infecciones.

Otras causas de deformidad de la muñeca que pueden simular DDM son la anemia de células falciformes, tumores y raquitismo ${ }^{(17)}$.

Cabe destacar que la DDM incluye una variedad de deformidades de la muñeca(18). DDM clásica: Subluxación dorsal del cúbito distal. La más frecuente. DDM invertida: Subluxación palmar del cúbito distal. Carpo en Chevron: La muñeca se mantiene alineada, con pequeña o nula deformidad. El hueso semilunar se impacta severamente en el radio distal. Es la variante más dolorosa.

Finalmente, se debe diferenciar la DDM de la enfermedad de Madelung o lipomatosis simétrica benigna, caracterizada por acumulación lipomatosa subcutánea en la región cervical, torácica superior y miembros superiores ${ }^{(19)}$.

\section{Discusión}

La DDM puede ser vista en radiografía simple, TC o RM, siendo el primer método diagnóstico suficiente en la mayoría de los casos.

Aunque no hay certeza sobre la prevalencia de la DDM, en radiología musculoesquelética constituye un hallazgo relativamente frecuente.

A pesar de considerarse una patología de la adolescencia, es importante considerarla en todos los grupos etarios. Así, en la población adolescente el radiólogo puede plantear el diagnóstico antes de 
que se establezca una deformidad más severa, permitiendo al clínico plantear opciones terapéuticas que permitan detener o revertir el progreso de la enfermedad, cambiando el pronóstico de estos pacientes. Por otra parte, aunque en adultos y ancianos puede presentarse en forma aislada, la correcta interpretación de sus hallazgos orienta al clínico a descartar un origen hereditario en base a estudios genéticos, con un potencial impacto en las generaciones posteriores en base a consejerías.

\section{Bibliografía}

1. França E. Congenital deformities of the upper limbs. Part III: Overgrowth; Undergrowth; Streeter and others. Rev Bras Ortop 2013; 48(2): 121-125.

2. Field JS, Rizzo M. Madelung Deformity With Prior Distal Radius Fracture: A Case Report. Am J Orthop 2007; 36(6): E91-E93.

3. Muzzo B, Castillo D, Burrows R. Discondrosteosis de Léri-Weill. Rev Chil de Pediatr 1985; 56(3): 184-187.

4. Dinamarca JL, Ávila A, Celis E. Deformidad de Madelung y discontrosteosis de Lèri-Weill: Revisión y reporte de serie familiar de adultos mayores chilenos. Bol Hosp Viña del Mar 2011; 67(3-4) - 2012; 68(1): 36-41.

5. Yang JH, Sohn YH, Ko SY et-al. Anthropological analysis of Koreans using HLA class II diversity among East Asians. Tissue Antigens 2010 Oct; 76(4): 282-288.

6. Lovell W, Winter R, Morrissy R et-al. Lovell and Winter's pediatric orthopaedics. Lippincott Williams \& Wilkins (2006).

7. Dannenberg M, Anton JL, Speigel MB. Madelung's deformity: consideration of its roentgenological diagnostic criteria. Am. J. Roentgen and Rad. Therapy Nucl Med 1939; 42(5): 671-676.

8. Greenspan A. Radiología de huesos y articulaciones. Capítulo 32 Anomalías de las extremidades superiores e inferiores, Deformidad de Madelung $2^{\mathrm{a}}$ Ed. Editorial Marban 2006; Pp. 891-893.
9. Langer LO. Dyschondrosteosis. A hereditable bone dysplasia with characteristic roentgenographic features. Amer J Roentgenol 1965; 95: 178.

10. McCarroll HR Jr, James MA, Newmeyer WL 3rd, Manske PR. Madelung's deformity: diagnostic thresholds of radiographic measurements. J Hand Surg Am 2010 May; 35(5): 807-812.

11. McCarroll HR, James MA, Newmeyer WL 3rd, Molitor F, Manske PR. Madelung's deformity: quantitative assessment of x-ray deformity. J Hand Surg Am 2005 nov; 30A1211-1220.

12. McCarroll HR, James MA, Newmeyer WL 3rd, Manske PR. Made- lung's deformity: quantitative radiographic comparison with normal wrists. J Hand Surg Eur 2008 oct; 33B: 632-635.

13. Vickers D, Nielsen G. Madelung's deformity: surgical prophylaxis (physiolysis) during the late growth period by resection of thedyschondrosteosis lesion. J Hand Surg Br 1992 aug; 17(4): 401-407.

14. Stehling $C$, Langer M, Nassenstein I, Bachmann $\mathrm{R}$, Heindel W, Vieth V. High resolution 3.0 tesla $\mathrm{mr}$ imaging findings in patients with bilateral madelung's deformity. Surg Radiol Anat 2009 aug; 31(7): 551-557.

15. Green DP, Pederson MD, Hotchkiss RN, Wolf SW, editors. Green's operative hand surgery. James MA, Bednar M. Deformities of the wrist and forearm. 5th ed. Philadelphia: Elsevier Churchill Livingstone 2005; pp. 1469-1479.

16. Golding JS, Blackburne JS. Madelung's disease of the wrist and dyschondrosteosis. J Bone Joint Surg Br 1976 aug; 58(3): 350-352.

17. Henry A, Thorburn MJ. Madelung's deformity. A clinical and cytogenetic study. J Bone Joint Surg Br 1967 feb; 49(1): 66-73.

18. Herdman RC, Langer LO, Good RA. Dyschondrosteosis. The most common cause of Madelung's deformity. J Pediatr 1966 mar; 68(3): 432-441.

19. Iglesias L, Pérez-Llantada E, Saro G, Pino M, Hernandez JL. Benign simmetric lipomatosis (Madelung's disease). Eur J Intern Med 2000; 11(3): 171. 